\title{
Multi Agent Systems combined with Semantic Technologies for Automated Negotiation in Virtual Enterprises
}

\author{
Koppensteiner Gottfried ${ }^{1}$, Merdan Munir ${ }^{1}$, Lepuschitz Wilfried ${ }^{1}$, \\ Moser Thomas 2 and Reinprecht Constantin ${ }^{3}$ \\ ${ }^{1}$ Vienna University of Technology, Automation and Control Institute \\ ${ }^{2}$ Vienna University of Technology, Inst. of Software Development \& Interactive Systems \\ ${ }^{3}$ Vienna Institute of Technology, Department for Information Technology \\ Austria
}

\section{Introduction}

In today's competitive global markets, no company can be successful without effectively managing its supply chain. Procurement is a crucial process and it accounts for more than half of the enterprises' sales volume. However, various problems have been identified in the past studies in this process: paper-based purchasing cycles and lack of automation, lack of intelligent advice tools for finding suitable suppliers, as well as difficulties to evaluate the performance of the suppliers (Lee et al. 2007). Appropriate supply chain organization will help companies to resolve conflicts, to reduce logistic lead times and total costs, to increase profits and to become more competitive on the market (Jain et al. 2009). Recent developments of electronic means for communication and collaboration between business partners led to the emergence of electronic negotiation as an alternative to manual contracting. The interest in e-negotiation is motivated by its potential to provide business partners with more efficient processes, enabling them to gain better contracts in less time (Rinderle and Benyoucef 2005). E-negotiation should allow automated or semi-automated processing of the information and transactions which take place between two companies and thus reduce costs and increase speed. However, by increasing the degree and the sophistication of the automation on both sides, commerce becomes much more dynamic, personalized, and context sensitive ( $\mathrm{He}$ et al. 2003). A major challenge in distributed software development is the integration of knowledge scattered over processes, tools and people to facilitate an effective and efficient coordination of activities along the development lifecycle. The EU research FP7 focuses on a key strategic research challenge ${ }^{1}$ in the area of semantic systems/technology development to deal with the "growing load and diversity of information and content" which particularly applies to software engineering projects where semantic support for data analysis and reasoning would improve data and knowledge exchange among distributed project teams and promises more effective collaboration.

\footnotetext{
${ }^{1}$ http://cordis.europa.eu/fp7/ict/content-knowledge/fp7_en.html
} 
Due to the high level of complexity and heterogeneity in these environments, traditional electronic commerce technologies have difficulties to support automated negotiation. The agent approach offers a convenient way to deal with complexity, autonomy and heterogeneity by providing the most natural means of representing the distinct individuals and organizations, suitable abstractions, the ability to wrap legacy systems and flexibility for organizational structure changes (Jennings 2001). The benefit of agent technology in contrast to the commonly used mathematical programming is that it is more versatile and can easily capture the qualitative as well as the quantitative nature of a complex technical system (Alvarado et al. 2007). The agents, each having individual goals, abilities and authorities, make decisions on behalf of their principals negotiating with each other, buying as well as selling goods or services and trying to achieve the best possible results. In this context, autonomous software agents are cited as a potentially fruitful way of approaching ecommerce automation (Kowalczyk et al. 2002). Nevertheless, traditional negotiation approaches pose a number of constraints on the type of interactions that can take place among agents, allowing only agents identified in advance to participate in a negotiation and predeterminating the protocols to be used (Tamma et al. 2005). Besides, most of the used MAS are designed with a single negotiation protocol explicitly hard-coded in the agents resulting in an inflexible environment able to accept only agents designed for it (Preist et al. 2002). Moreover, typical semantic gaps exist in the exploitation of current agent systems considering the fact that mostly just syntactic matching is used for detecting equalities between exchanged data during the communication between agents.

To allow communication among heterogeneous systems, there must be an agreement on both the syntax and the semantics of the exchanged messages or of any other type of interaction. The syntax defines the structure of a language, i.e., a grammar typically in a form of rules that govern the structure of sentences. Semantics refers to the aspects of meaning as expressed in the language, i.e., the sense of language elements and their combination, including the relation of these elements to the real world. Ontologies are a way of representing concepts and relationships embedded in the negotiation environment such that they are semantically meaningful to a software agent, ensuring that agents refer to the same object (Beam and Segev 1997). Ontologies can be used to describe the semantics of the information sources and make the contents explicit, thereby enabling the integration of existing information repositories. The negotiation ontology, that specifies basic terms an agent has to share in order to enable understanding of the used concepts and conditions under which the interaction between agents takes place, is defined by Tamma et al. (Tamma et al. 2002). Besides, the negotiation ontology is used to describe interaction protocols related to the dynamic Cross-Organizational Workflow context in order to permit the different agents to dynamically select at run-time a given negotiation protocol during the opening of a negotiation step (Bouzguenda et al. 2008). However, the overall efficiency of the negotiation process is, besides the general "understanding", strongly influenced by different attributes of the deal (quality of product/service, availability, risk, delivery time, etc.) whose consideration/non-consideration can significantly influence the agent's results. Besides, up to now, most of the research done on automated negotiation considers models where the price is the unique strategic dimension (Renna and Argoneto 2010).

A decision support system (DSS) is required which is able to check multiple attributes of a deal and by merging them suggests suitable solutions (David et al. 2006). In this work we combine a multi-agent framework with ontology-driven solutions to support and automate 
the procurement process. The resulting Semantic-enabled Decision Support System (SemDSS), which is composed of agents with different goals and tasks organized in a suitable architecture, is able to simultaneously consider multiple attributes of a negotiation and assists users in the decision-making process. In our approach, the agent is an autonomous semantic entity having specific tasks and knowledge about its domain of application in the procurement process, about strategies, which can be used to achieve a specific goal during the procurement process, as well as about (other) relevant agents involved in the DSS. Considering that the ontological framework is the key for agents to "understand" the process and to link the context of data, process or services within or between entities, we introduce a semantic foundation to bridge relevant semantic gaps in order to radically improve the automated data processing in semantically heterogeneous environments, i.e., information from different users or open source communities with their own API standards. This semantic foundation is based on a semantic model, describing the information to be integrated as well as artifacts in a way, which is both human-readable as well as useful for machine-based automated parsing and logic reasoning. The model is supported by automated semantic annotations of artifacts, based on their natural language descriptions.

\section{Motivation study}

The Automation and Control Institute of the Vienna University of Technology has conducted the study "Roadmap for the movement of SMEs towards semantic knowledgebased systems" within the framework of a project financed by the Vienna Economics Chamber. This study analyzed current state of the art and issues related to the application and capabilities of semantic technology in more than 100 Austrian companies, which are employed in various sectors (information and consulting, transport, tourism, industry, bank, etc.). The results of this study have shown that almost $40 \%$ of companies have difficulties in the data exchange with other business partners, since data is not represented in a uniform format. $51 \%$ confirm that the amount of manual data processing is too high. For almost $62 \%$, the optimization of business processes and improving cooperation with business partners would be the main reason to introduce a new IT system (Merdan et al. IN PRESS).

\section{State of the art}

\subsection{Agent-based decision support systems}

Decision support systems have been used by negotiators to support individuals in negotiations. They are user-oriented, because they help users to understand and formalize their objectives and preferences. Moreover, they are problem-oriented because they help users to understand the problem structure, search for solutions, and conduct sensitivity analyses (Kersten and Lai 2007). Early DSS work advocates the development of such systems to allow users to quickly develop them application-specific (Bui and Lee 1999). Multi-agent technology is a superior solution that can enhance a system's connectivity, extensibility, reusability, and manageability. Intelligent agents are goal-oriented, collaborative, flexible, self-starting, intelligent and interactive (Turban et al. 2005). These characteristics of agents ensure the application of multi agents in DSS to be successful (Zhang 2009). Some reports on the application of agent technologies for DSS exist in literature. Ai et al. presented a distributed marketing decision support system (Ai et al. 2004). Houari and Far presented a novel architecture based on multi-agents technology to 
support information and knowledge extraction over distributed data sources in order to use them in the decision making process (Houari and Far 2004). Zhang and Xi present a decision support system framework for the partner choice and measurement (Zhang and Xi 2005). Neubert et al. proposed a software agent, capable of conducting an automated negotiation in order to assist the human decision-maker in an environment consisting of small independent units (Neubert et al. 2004). Wang et al. presented the architecture of Weboriented Warfare Command DSS (Wang et al. 2005). Zhang and Yu reported on the bidding decision-making for power suppliers (Zhang and $\mathrm{Yu}$ 2006). Puigjaner et al. conducted a research in the context of supply chain in the chemical process industry (Puigjaner et al. 2008). However, in most of the cited papers, the considered solutions are pretty much custom oriented and the negotiation process takes just one parameter (the price) or only one item into account. Moreover, the presented approaches lack solutions that support interoperability when applied in a heterogeneous environment. Having identified the need to extend current DSS approaches to effectively and efficiently facilitate the integration of data from heterogeneous systems, the research on semantics provides a range of very useful concepts to build a strong semantic foundation for DSS.

\subsection{Ontologies for integrating domain, project and tool data}

Ontologies can represent domain vocabulary, i.e., the conceptualizations that the terms in the vocabulary are intended to capture (Chandrasekaran et al. 1999, Davies 2006). Ontologies have been used in IT for a range of objectives: for clarifying the knowledge structure, for reducing conceptual and terminological ambiguity and for enabling knowledge sharing extensions to typical IT projects (Calero et al. 2007, Hepp et al. 2007, Pastor et al. 2008). Typical objectives for ontology usage include a) the declarative specification of a system; b) the support for manual or semi-automated consistency checks; c) the improvement of the documentation and a reduction in the efforts needed for maintenance; d) the reuse of aspects between different domains or tasks; and e) the acquisition and storage of knowledge, e.g., with reasoning (Happel and Seedorf 2008, Baclawski et al. 2001).

\subsection{Semantic integration of data from heterogeneous sources}

Providing integrated access to multiple heterogeneous sources in information systems raises challenging cooperation and interoperability issues (Bergamaschi et al. 1999): firstly, how to determine if the sources contain semantically related information, i.e., information related to the same or similar real-world concept(s), and secondly, how to handle semantic heterogeneity to support integration and uniform query interfaces. The successful use of the ontologies concept within modern data integration and business intelligence techniques and technologies has been reported particularly within heterogeneous domains where the use of common meta-data, services and processes seems most valuable (Fonseca et al. 2000). An ontology enables information from one resource to be mapped accurately at an extremely granular level to information from another source. The ontology provides the common vocabulary for the data integration - showing the preferred names for a concept, and the synonyms and properties associated with this concept. This enables the forward-looking integration by collecting data using names that are already well understood rather than ones that might not be shared widely throughout the organization. This makes the assimilation of new data easier and quicker, and facilitates communication between groups (Eilbeck et al. 
2005). Furthermore, the ontology can grow over time as new data become available, new links are continually being made and new knowledge assimilated in the ontology (Kiryakov et al. 2004). Moser et al. (Moser 2009; Moser et al. 2010) introduced the Engineering Knowledge Base (EKB) framework as a semantic web technology approach for addressing challenges coming from data heterogeneity that can be applied for a range domains, e.g., in the production automation domain and also software engineering. Further, Biffl et al. (Biffl et al. 2010) used the approach for solving similar problems in the context of open source software projects, in particular, frequent-release software projects.

\subsection{Tool support for semantic annotation}

Semantic annotation is a specific metadata generation and usage schema targeted to enable new information access methods and extend existing ones. Annotation schemas are often based on the understanding that the named entities mentioned in documents or artifacts constitute important parts of their semantics. Furthermore, using different sorts of redundancy, external or background knowledge, those entities can be coupled with formal descriptions and thus provide more semantics and connectivity to other resources (Michelson and Knoblock 2007). Ontological structures may give additional value to semantic annotations. They allow additional possibilities concerning the resulting semantic annotations, such as reasoning. Furthermore, an ontology directs the attention of the annotator to a predefined choice of semantic structures and therefore gives some guidance about what and how items residing in the documents may be annotated (Staab et al. 2001).

There are a number of tricky issues with providing semantic annotation: First of all, the semantic annotation task does not adhere to a strict template structure, such as Dublin Core to name one of the more sophisticated ones in use. Rather it needs to follow the structure given by schema definitions that may vary with, e.g., domain and purpose. Semantic annotations need to be congruent with ontology definitions in order to allow the advantages we have indicated above. Secondly, semantically interlinked metadata is labor-intensive to produce and, hence, expensive. Therefore duplicate annotation must be avoided. Because semantic annotation is a continuous process in a distributed setting, there are several sources for duplication. Thirdly, purely manual annotation is very expensive. Therefore, only very valuable information will be annotated and it is necessary to help the human annotator with his task. What is needed is support for automatic - or at least semiautomatic - semantic annotation of information. Finally, there is a lack of experience in creating semantically interlinked metadata. It is not clear how human annotators perform in total and, hence, it is unclear what can be assumed as a baseline for the machine agent (Cimiano et al. 2004).

In the context of distributed projects automated semantic annotation tries to identify the semantic meaning of natural language terms or descriptions of artifacts by parsing the terms and analyzing the interrelationships between noun groups and verbal groups, based on a set of semantic categories defined for a certain project. By now, semantic annotation is primarily a manual task that needs additional time and efforts, resulting in reluctance of the project stakeholders to fulfill the task if the added value of their action is not clearly visible. The semantic annotations can later on be used for the derivation of new or à-priori unknown facts using semantic reasoning mechanisms provided by ontology reasoners (e.g., RACER $^{2}$, Pellet ${ }^{3}$ or $\left.\mathrm{FaCT}^{4}\right)$. Using the capabilities provided by these reasoners, it is possible

\footnotetext{
2 http:/ / www.racer-systems.com/
} 
to detect new or unknown relationships between software artifacts, errors or faults in the consistency of the artifacts stored/created by the different tools, as well as to effectively perform change impact analyses by exploiting the information on relations between the modeled individuals/artifacts (Michelson and Knoblock 2007).

Because meaningful sentences are composed of meaningful words, any system that hopes to process natural languages as people do must have information about words and their meanings. This information is traditionally provided through dictionaries, and nowadays machine-readable dictionaries are widely available. But dictionary entries evolved for the convenience of human readers, not for machines. WordNet ${ }^{5}$ provides a more effective combination of traditional lexicographic information and modern computing. WordNet is an online lexical database designed for the usage under program control. English nouns, verbs, adjectives, and adverbs are organized in sets of synonyms, each representing a lexicalized concept. Semantic relations link these synonym sets (Miller 1995).

\section{System architecture}

\subsection{SemDSS architecture}

The agents' architecture for the SemDSS System is derived from a general automated negotiation framework, where two or more entities try to reach agreement on one or more matters of common interest. The system is composed of three main parts (see Figure 1): (i) the Auction Framework, (ii) the Agent based Negotiation System with the Decision Support Algorithm (DSA) and (iii) the Decision Support System for Supplier Ranking.

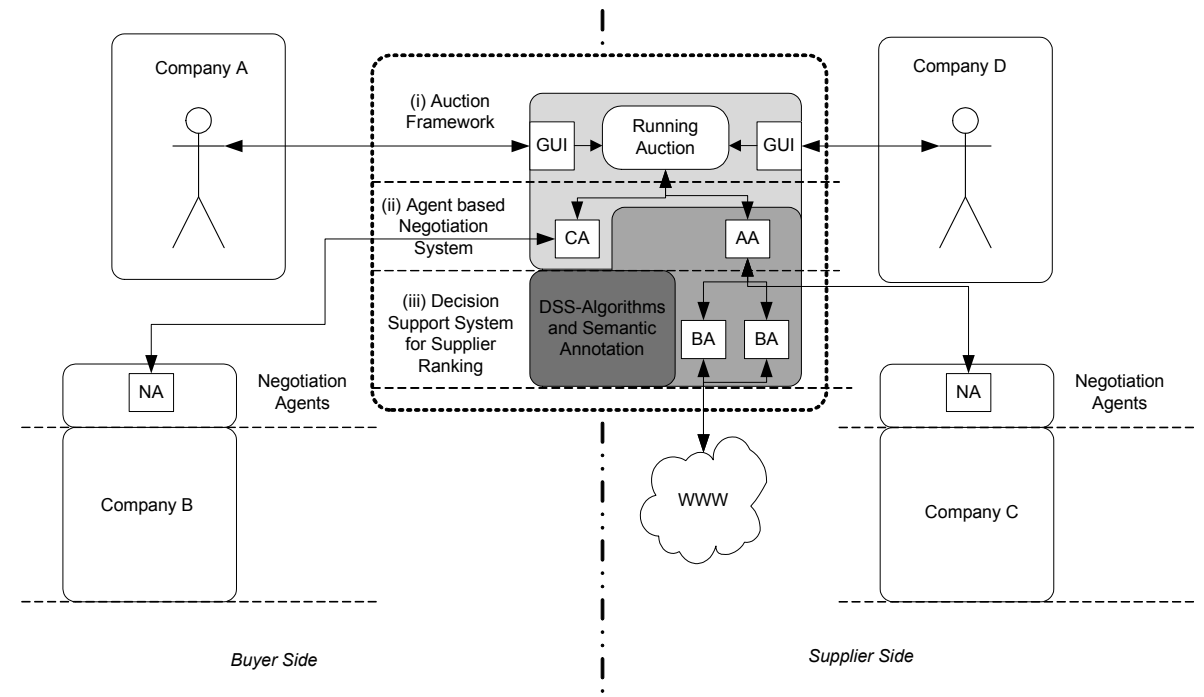

Fig. 1. SemDSS Overview

\footnotetext{
${ }^{3}$ http:/ / pellet.owldl.com/

${ }^{4} \mathrm{http}: / /$ jade.tilab.com/

${ }^{5} \mathrm{http}: / /$ wordnet.princeton.edu/
} 
The Auction Framework defines the environment required to enable an automated negotiation. It specifies the responsibilities and behaviors of involved entities and handles all necessary activities during the negotiation process allowing one entity (a purchaser) to start the negotiation as well as other ones (suppliers) to participate in it and place bids. The Agent based Negotiation System and related architecture is already developed and successfully tested at ACIN and consists of a Contact Agent (CA), responsible for the first contact of a user (buyer or seller) with the Auction Framework and an Auctioneer Agent (AA), which is upgraded in the presented system. Additionally, at each company there could be a Negotiation Agent (NA) which represents the user/company. The only tasks of this NA are to compete in auctions if the company wants to buy something or to inform the Auction Framework if the company wants to start an auction in order to sell a product or a service. The auctions themselves are running on the central Auction Framework, which is placed on its own server. The company's NAs are running on the company's server and can interact with other agents of that company. The CA on the negotiation system is just responsible for the first requests from the company's NA and for creation of the AA for each auction. The negotiations are running directly between the NAs on the company's sides and an AA on the Auction Framework. The AA, which collects information about every auction and stores them in its knowledge base using semantic annotation methods to enhance the information quality, is also used as the interface between the Agent based Negotiation System and the Decision Support System for Supplier Ranking. The AA's further responsibilities are associated with the system's supplier ranking activities and will be more elucidated. This system is using standardized protocols and messages based on ontologies and is able to run different kinds of auction types. It integrates a special Decision Support Algorithm, which is able to select the most profitable auction type for a specific good on the buyer side as well as to propose the most suitable strategy for placing bids on the seller side (Koppensteier et al. 2009a). It has been shown that companies which are using the Auction Framework and the Agent based Negotiation System, as described in (Koppensteiner et al. 2009), could enhance their agility and improve interoperability when forming virtual enterprises.

\subsection{Decision Support System for Supplier Ranking}

The Decision Support System for Supplier Ranking, as a third part of the SemDSS, is derived from the normal auction process steps (see Figure 2). Normally, the first step in an auction process is to (i) start the auction. A buyer introduces information into the auction systems concerning the wanted goods, about the expected performance (such as delivery, lead-time, quality, price, or some combination of these variables) as well as about the start and end of an auction. The AA, which is automatically created, has a special behavior to store all the given information in its knowledge base using a semantic annotation method. After that, the AA has to (ii) wait for the bids of possible suppliers and to monitor the auction. It creates a Bid Agent (BA) for each incoming bid with semantically prepared information about the auction properties and the supplier. The bids are (iii) evaluated by the BA according to correctness and reliability of the vendor and forwarded further to the AA. In the case of a misplaced bid, the BA informs the AA and determinates itself. If more than one supplier places a suitable bid, the AA has to rank the bids.

At some point, companies must reevaluate or even eliminate suppliers that do not fit well with the buyer. This can be a huge task, depending on the number of suppliers and the obtained information. In order to do this, internal and external information will be collected and evaluated. Nevertheless, many large companies are divided into units, each with a 
separate purchasing sector. Sharing information across units can occur through informal meetings, strategy development sessions, newsletters, or the development of a comprehensive database containing information about potential supply sources. Internal sources, even those from diverse business units and their further experience with the suppliers, can provide a great deal of information about potential supply sources.

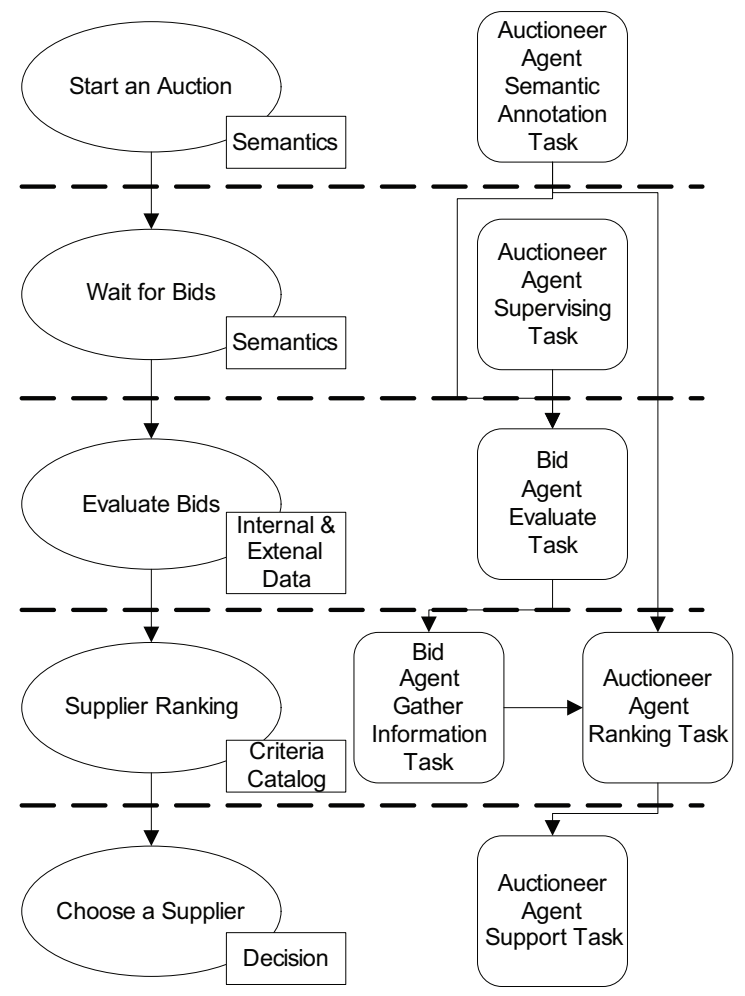

Fig. 2. Overview of the DSS-based auction process

The gathering of internal information and their representation in the knowledge base is one important task of the BA. It uses an ontology-based approach where different kinds of matchmaking strategies are combined together to provide an adaptive, flexible and efficient knowledge discovery environment (Horrocks and Li 2004, Mäkelä 2005, Bianchini et al 2008). Moreover, buyers and sellers are increasingly using the internet to help locate potential sources that might qualify for further evaluation. However, a major problem with searching in the Web today is that data available in the Web has little semantic organization beyond simple structural arrangement of text, declared keywords, titles, and abstracts (Luke and Rager 1996). The BA is using diverse semantic-based search algorithms to get information about the supplier in the World Wide Web (Sycara et al. 2003, Rao and Su 2004, Milanovic and Malek 2004). The content of Web pages is expressed by referring to ontologies, which provide a conceptual model to interpret the content (Bruijn et al. 2009). After collecting information about potential supply sources, the BA must begin to filter and consolidate the information. The information has to be arranged according to a self-defined criteria list, 
defined at the start of the auction. This especially, due to the amount of data that are being gathered by the BA about different suppliers. At the end, the AA gets all the collected and arranged information from the different BAs and performs its ranking task. The decision about the ranking is documented and reported to the buyer, which is also done by the AA. The presented Decision Support System for Supplier Ranking enhances the existing system with the BA abilities, which are used for evaluating the bids' correctness and for finding as much information as possible about the supplier. The BA agent is equipped with related behaviors to gather information, filter information, arrange them in its knowledge base and learn from this information. As information collector, this agent identifies eventual file locations about the supplier or an auction user as well as the desired amount of information by enquiring the AA. It gets the information about the buyers' preferences such as timeconstraints, suppliers' reliability, and quality of products. If necessary, it uses also the sources like the Credit bureau ${ }^{6}$ or similar sources to be able to offer reliable information.

\subsection{Semantic annotation integration}

The Semantically-enabled DSS provides a framework and integrated methodology consisting of a) the semantic model (describes domain, suction, suppliers' knowledge); b) tools for adapting, maintaining, and analyzing knowledge bases; c) semantic services (connection of the semantic model to the auction system repository, semantic tools) and user interfaces to view and manipulate the semantic model; and d) processes and methods for adapting SemDSS to the auction platform, setting up auctions with SemDSS. The first goal of SemDSS is to provide semantic foundations for auctions to support semantic data analysis. The semantic foundation layer is depicted in the lower part of Figure 3.

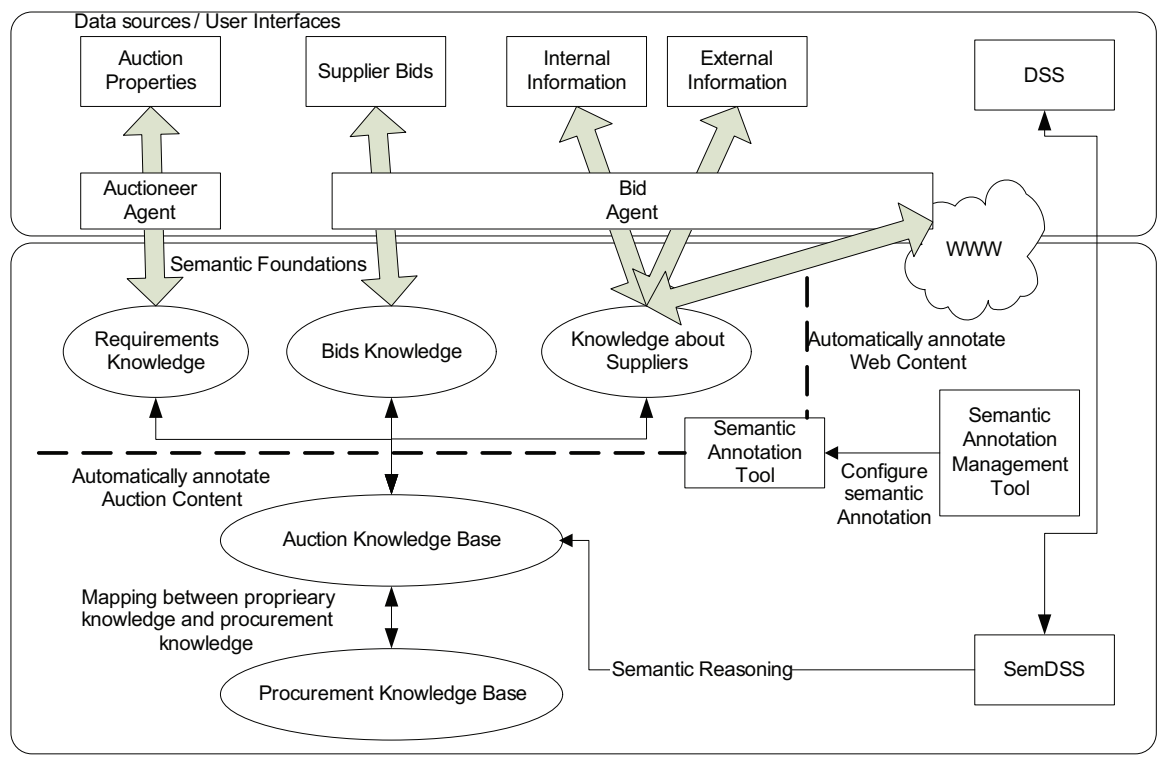

Fig. 3. Semantic Foundations for SemDSS

${ }^{6}$ http://www.ksv.at/KSV/1870/ 


\subsection{Negotiation ontology}

It is important for each company to define its own point of interest in a negotiation and to be able to estimate other participants. Due to the complex and dynamic nature of the VE it is hard to capture all related concepts in a persistent ontology. It is much simpler to isolate the ontology part that is only related to data exchange and communication processes associated with it. In this context an ontology was defined that offers the representation and semantics of data about negotiation and presents the link to all other concepts in the company (see Figure 4). This negotiation ontology will include a description of basic company internal concepts (order, user, product/service, interfaces, etc.).

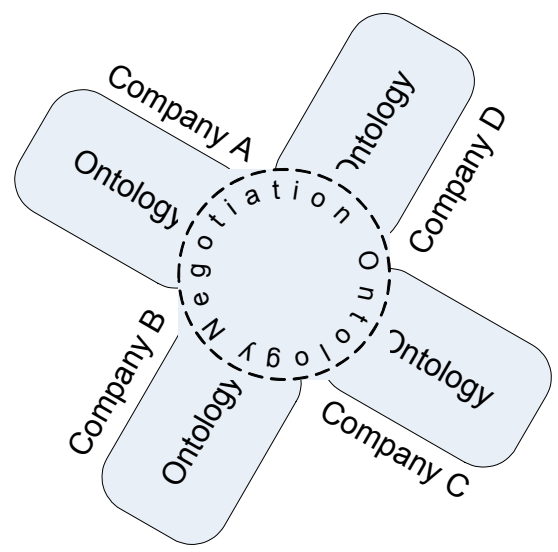

Fig. 4. Negotiation Ontology

An agent that has to make related conclusions should have knowledge about all related concepts within the domain of application. It has to know the users' economic profile (value of product/service to be sold/bought, its total cost including shipment and production, quality, etc.) and its complexity and risk profile (size of supply market, uniqueness of product, availability, etc.). Agents within the SemDSS are also externally influenced in terms of their relationships with other participants, which depend on each other considering goals to be achieved or tasks to be performed. Goals could be to buy at the lowest possible price or at the highest possible quality. Tasks could be a participation in a negotiation or the supervision of an auction. Additional factors possibly influencing negotiations with other agents could be the number of possible sellers/buyers, communication and information sharing, reputation, their reliability and the quality or quantity of the product/service. To be able to reach its goals and accomplish the tasks as good as possible a decision about an auction or strategy has to be done according to all these attributes. Consequently, it is necessary to model and represent all these concepts such as the different possible auction types, negotiation processes and rules as well as dependencies between agents and partners in the supply chain.

The negotiation ontology (see Figure 5) ensures that the agents assign the same meaning to the symbols used for messages during the negotiation process. Moreover, an ontology supports not only the communication between agents but also gives the possibility for agents to reason about the domain of application. Furthermore, it enables that agents participate in negotiations without prior knowledge of the negotiation mechanism and the 
ability to exchange knowledge about it reduces the amount of knowledge hardcoded in the agents (Tamma et al 2005). The presented ontology offers a representation of the general framework in detail to an agent.

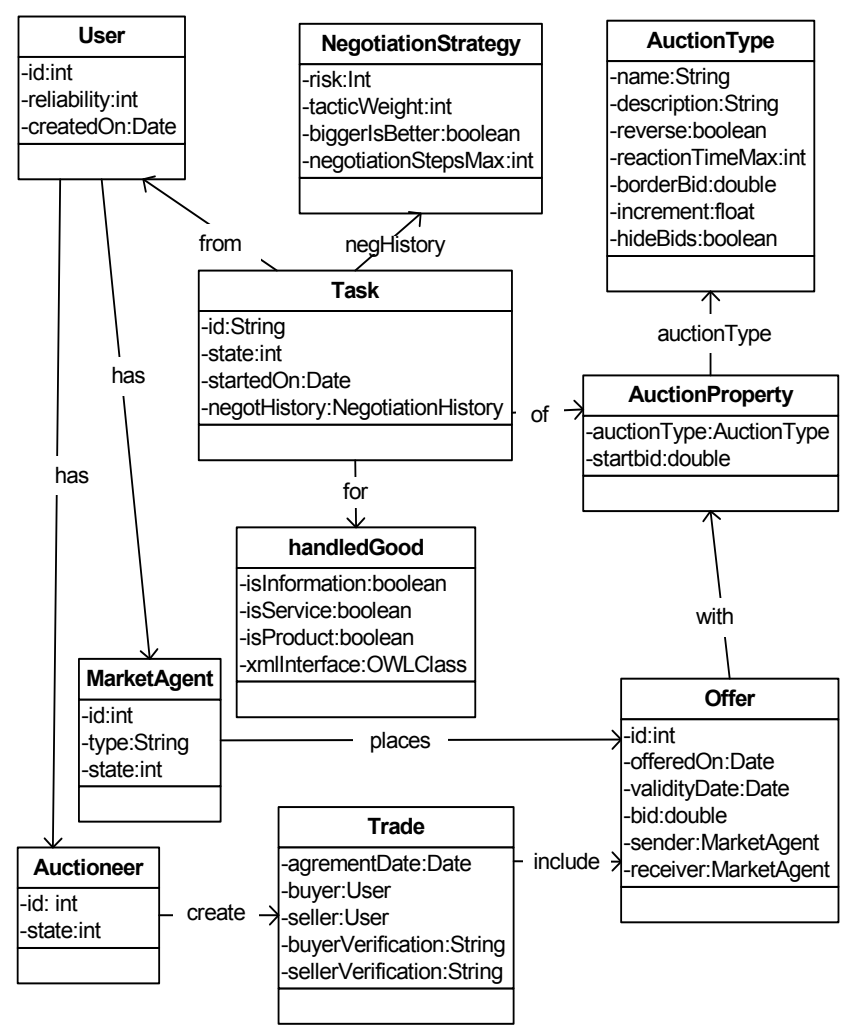

Fig. 5. Overview of the Negotiation Ontology

\section{System implementation}

Our MAS is based on the Java Agent Development Environment ${ }^{7}$ (JADE) framework. The framework is used to establish the distributed agent network placed on different platforms. We also use this framework in order to support the exchange of ACL-messages between the distributed agent platforms. We used the Jess expert system shell ${ }^{8}$ (JESS), in order to implement each agent's rule-based behavior. Every piece of information (i.e., every instance) of the ontology is mapped into a JESS fact. The JESS engine requires these facts to match them with the if-parts of rules and to execute appropriate rules. Each agent of our MAS is equipped with the negotiation ontology to share a common understanding within negotiations with agents of other platforms. The negotiation ontology can be seen as an add-

\footnotetext{
7 http://www.fipa.org/

${ }^{8}$ http:/ / herzberg.ca.sandia.gov/
} 
on to the companies' ontologies. We used Protégé 9 to develop an agent's knowledge base. Every NA is also equipped with a graphical user interface through which the user can compete in negotiations as well as to start new ones.

Figure 6 demonstrates, how the graphical user interface (JAVA), the knowledge ontology (Protégé), the agent's rule-based behavior (JESS) and the multi-agent framework (JADE) work together in order to send a message from one agent platform to another.

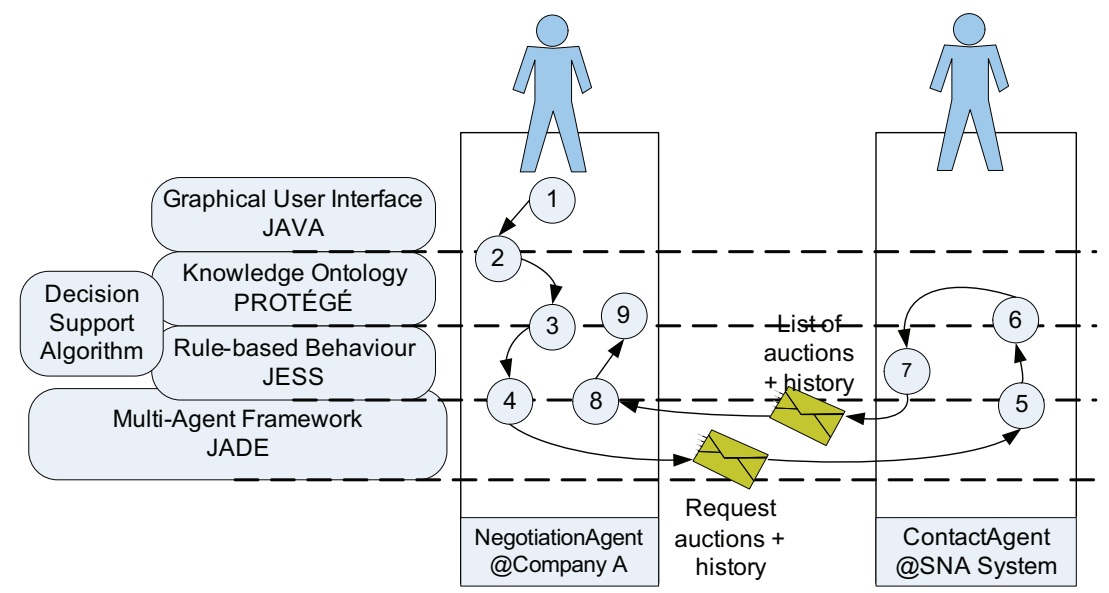

Fig. 6. A Negotiation Agent requests a list of auctions and history information

The simplified process to request negotiation and history information from the Contact Agent of the semantic negotiation system is described as following:

9. The graphical user interface (GUI) is created for the user. With this GUI the user can compete in negotiations as well as start a negotiation. In this example, the user wants to compete in an auction/negotiation because he wants to buy a specific merchandise/service. With the, the user can specify which merchandise/service he wants to buy and the user can also adjust his settings according to his maximum price as well as his price- and his quality-focus.

10. The information from the GUI is mapped to the ontology. The ontology represents the actual world of an agent. Each instance of the ontology is mapped as fact into the JESS fact base, which is part of the agent behavior. So it is possible that the agent can react on facts according to its rules.

11. Based on the facts from step 2, a rule to send a message to the CA of the Auction Framework requesting the list of available auctions and the history information for this merchandise will be executed.

12. The message from the NA to the CA is sent through the JADE runtime environment to the related CA of the semantic negotiation platform.

13. The CA receives the message and maps it to its knowledge base.

14. After mapping the message to the knowledge base, a rule will be executed which searches all available negotiations as well as the negotiation history for the merchandise, the NA wants to buy.

\footnotetext{
${ }^{9}$ http://protege.stanford.com/
} 
15. The CA sends the message with the list of negotiations and the history information back to the NA.

16. The NA receives this message and maps it to its knowledge base.

17. Based on the facts of step 8 , a rule will be executed which maps the information of the content of the message of step 8 to the NA's knowledge base. Another rule will be executed, which calls the Decision Support Algorithm. The DSA evaluates the possibly best auction according to the price- and the quality-focus of the user and the negotiation history for that merchandise/service.

Figure 7 is the continuation of Figure 6. It shows the simplified basic activities of the Negotiation Agent and the Auctioneer Agent in an auction or negotiation between companies.

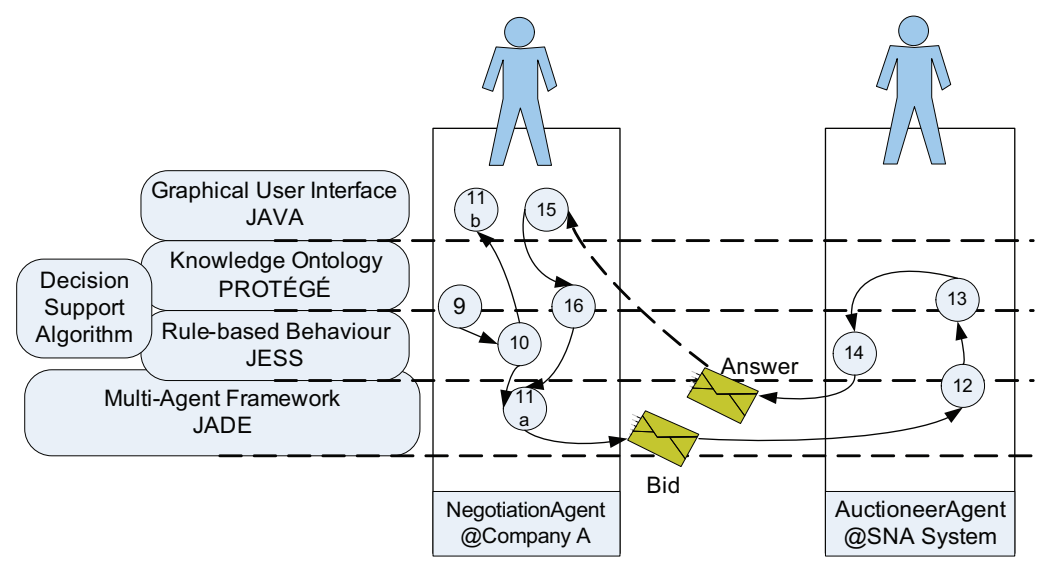

Fig. 7. The Negotiation Agent negotiates with an Auctioneer Agent

We will continue explaining with step 9 which is identical in both Figures 6 and 7.

9. The DSA evaluates the possibly best auction according to the price- and the qualityfocus of the user and the negotiation history for that merchandise/service.

After step 9, the NA would send a message to the AA in order to register for this auction. The AA would answer with a call for proposal (CFP) which is the starting point for the actual negotiation with bids and answers. Because the bid and answer messages between the NA and the AA are the most important ones, they will be described in the following:

10. The NA prepares a bid for the auction he joined.

11. Step 11 is split into two tasks:

a. The message containing the bid is sent through the JADE runtime environment to the related AA.

b. The bid is also shown in the GUI.

12. The AA receives this message with the bid and maps it to its knowledge base and starts the Bid Agent for the gathering of information about the seller.

13. The AA compares the bid with the current price of the auction and bids from other agents, as well as the information from the NA.

14. Based on the auction type, the AA sends information about the current state of the auction to all NA which join the auction.

15. The answer from the AA is shown in the GUI of the NA. 
16. According to the answer of the AA, the NA either places another bid or waits for bids of other agents and the end of the auction (if it currently has the highest bid).

This negotiation cycle goes on as long as the maximum price of a NA is not reached and as long as the runtime of the auction has not expired. If the NA places another bid, the cycle continues with step 11a.

\subsection{General use case concept}

In order to present our concept, we selected different companies with related products and service to build a VE. The major aspect here is that associated and related ontologies cover different concepts and workflows within these companies. Moreover, while such companies can be placed anywhere, the possibility exists that they use different words for the description of the same concepts or vice versa. The selected VE-concept is shown in Figure 8 .

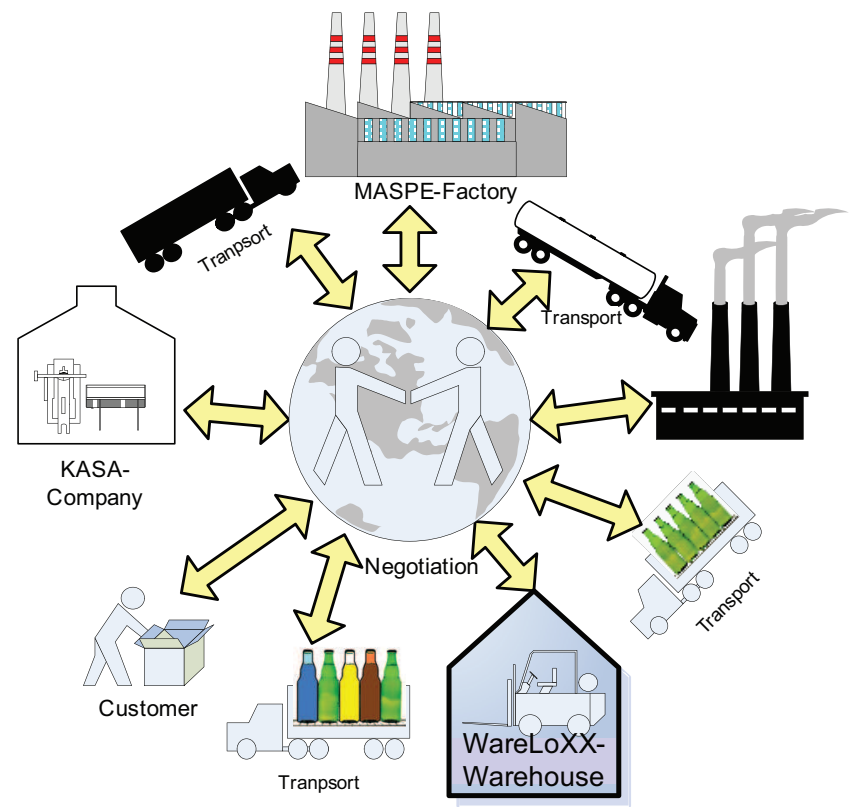

Fig. 8. Virtual Enterprise Concept

The following use-cases were separately analyzed and developed by four project groups:

- KASA-Ontology: A representation of a company for agent based assembly automation;

- MASPE-Ontology: An agent based batch processing factory for liquids;

- WareLoXX-Ontology: A warehouse system for the commitment of orders; and

- KABA-Ontology: A bottling plant for the filling of bottles combined with LiStoSysOntology.

The specified companies need to cooperate and negotiate with each other to be able to place products on the market. The usage of the negotiation ontology that links all the different ontologies of the different companies enables the determination of an equivalent or the semantically closest concepts. 


\subsection{Different companies - different ontologies}

In Figure 9 two different ontologies for the handling of the term "product" are shown. On the left side of the picture an ontology for batch processes is shown. The class Product serves as a unique naming class for a product (i.e., for instance a specific amount of a pharmaceutical product) by using an ID and refers to the class Recipe, which contains all required material resources (such as raw material) and operations to manufacture this product step by step. The concepts of certain classes, such as the concept of a batch or a recipe, are derived from the relevant standard IEC 61512 Batch Control10.One the right side a product is presented as a hierarchy of subassemblies and parts together with all their properties and relationship between them. Parts are defined as components, described by a set of attributes, properties, constraints and relations to other parts. Each company in the VE has the same ontology-bases and also their own independent ontology concepts, necessary for covering their internal workflow. The negotiation ontology is used to ensure the overall understanding during communication and to enable the mapping of external information and knowledge into an internal company representation.

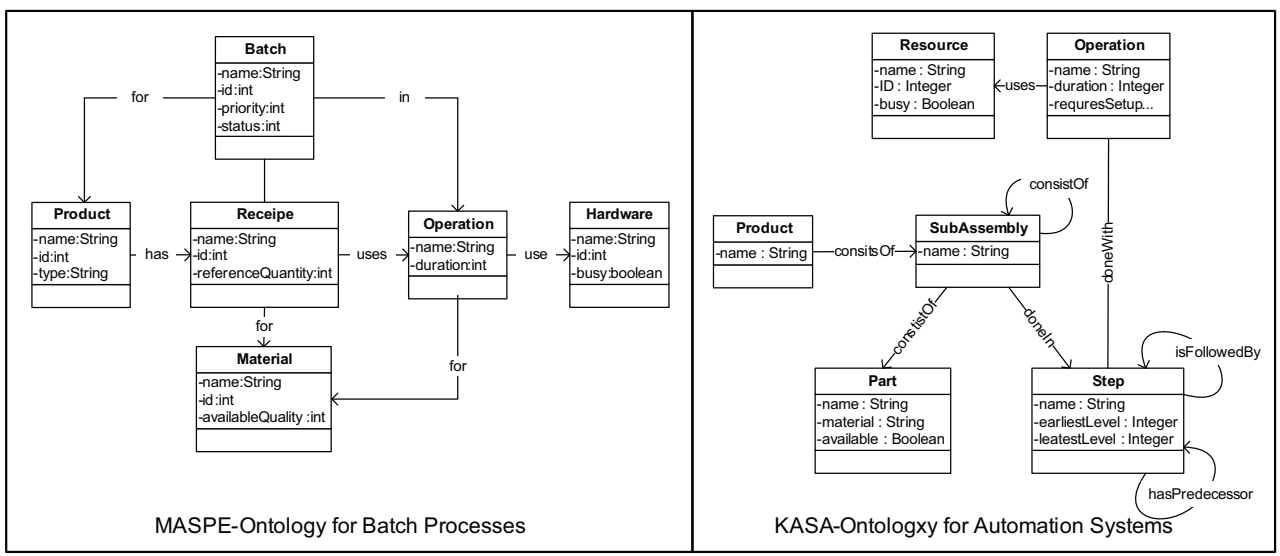

Fig. 9. Different Ontologies

We presented only a particular example the show the diversity between ontologies of different companies. Detailed examples can be found in (Koppensteiner et al. 2009).

\section{Evaluation}

\subsection{Evaluation of intelligent agent approach in negotiation systems}

This section will demonstrate the benefits of intelligent agents within the SemDSS framework as opposed to ordinary negotiation agents. The DSS negotiation agents have the ability to consider not only the price as the attribute for competing in negotiations, but also some other attributes such as quality, availability, etc. For the following two evaluations of our approach, negotiations with a start price between 30 and 70 and a runtime between 15 and 30 seconds were randomly generated. Besides the price and the runtime, all negotiations share the same attributes as quality, increment step or terms of payment. The

${ }^{10}$ IEC TC65, IEC 61512-1: Batch Control - Part 1: Models and terminology, International Electrotechnical Comission (IEC), 1997 
negotiation agents have the task to buy products from 10 different negotiations. Their maximum price varies randomly between 70 and 100 units and changes for each negotiation they participate in. Figure 13 shows a comparison between one intelligent agent (DSS_Agent, dashed line) and three ordinary negotiation agents (Agent_1, Agent_2, Agent3). The intelligent agent chooses in which negotiation to participate in, as well as which maximum price it should offer in order to buy the products at the lowest possible price. Furthermore, the agent uses a history analysis to get the mean average product prices of the past which helps it to choose a negotiation and a maximum price.

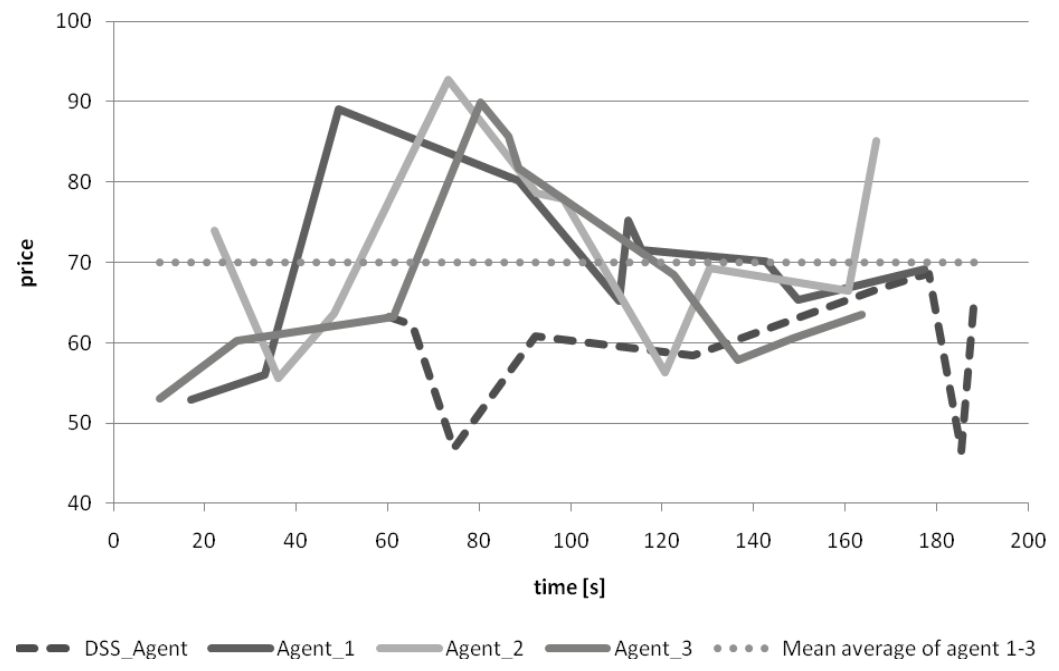

Fig. 10. Comparison between intelligent and ordinary negotiation agents

Figure 10 demonstrates that the intelligent DSS agent buys the wanted products at a cheaper price than all other agents. It might seem strange that the intelligent agent wins its first negotiation after 60 seconds compared with the other agents which win after about 10-20 seconds. This fact can be explained with the use of the DSA, the random generation of auctions as well as the random decision of a maximum price for a negotiation. The DSS agent decides to take a low maximum price for the first auctions and increases its maximum price for every lost auction. The DSA, which is used to evaluate in which negotiation the intelligent agent should compete in, currently does not consider if the products are needed urgently or not. An effective way to reduce the time until the intelligent agents wins its first negotiation would be to add a time factor (are the products needed urgently or not) to the DSA or simply to select a higher maximum price for the first auctions. The figure also shows that the mean average price of negotiations of the three ordinary agents (dotted line) is higher than each single negotiation of the intelligent agent.

In order to prove that the intelligent agent with the SemDSS framework gives advantages in negotiations, the evaluation shown in Figure 10 has been repeated 10 times and the mean average price of each negotiation agent for each repetition can be seen in Figure 11. The figure clearly demonstrates the advantage of an intelligent agent as opposed to ordinary negotiation agents. The DSS agent wins negotiations on an average with a $19.87 \%$ lower price than the other negotiation agents do. 


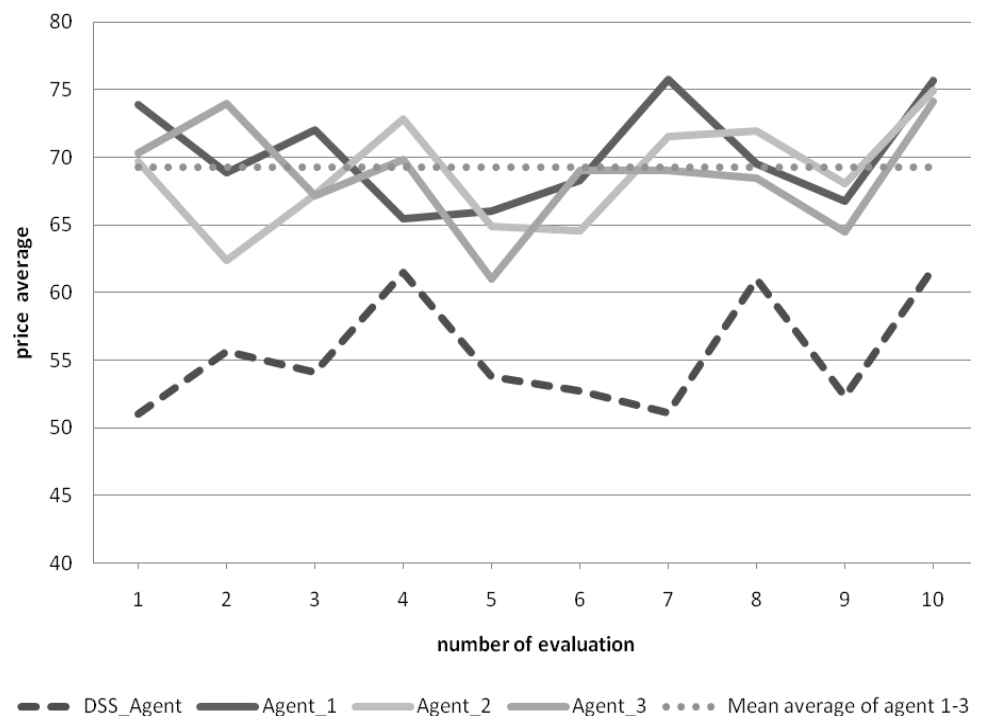

Fig. 11. Comparison between intelligent and ordinary negotiation agents

As already mentioned, the DSS negotiation agents are able to consider more than just one attribute of a negotiation. Figures 12 and 13 deal with a comparison between a pricefocused, a quality-focused and a price- and quality-focused DSS agent. The intelligent agent considers the price as well as the quality of products, weights and ranks them and chooses the best suitable negotiation according to the ranking. For the evaluation, the system created random negotiations with a quality between 1 and 10 and a random price between 20 and 60. Negotiations with products of higher quality tend to have a higher price than negotiations with products of lower quality. Each of the three agents will participate in negotiations as long as it wins 10 of them. To reach more significance, this process has been repeated 10 times and the mean average price and quality values of the negotiations an agent won can be seen in Figures 12 and 13.

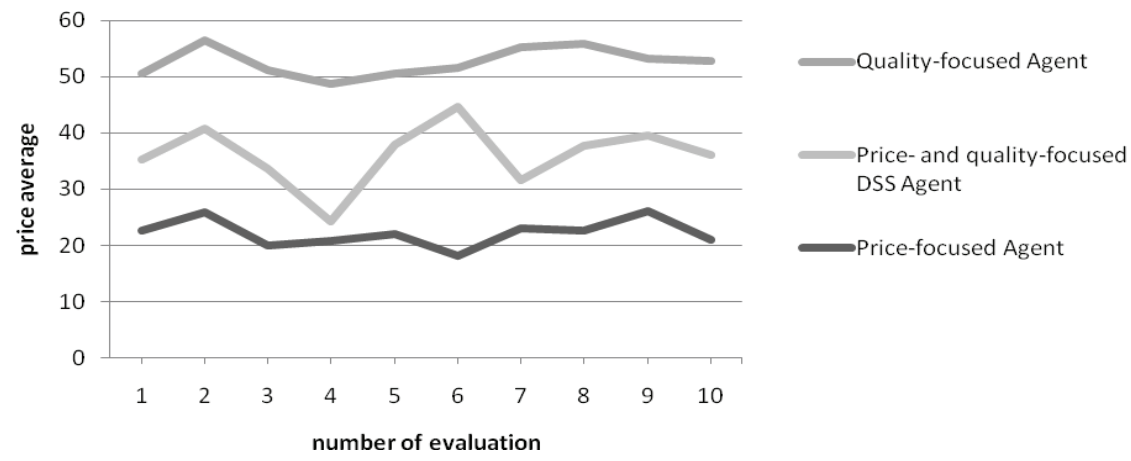

Fig. 12. Comparison between quality-, price-, and quality- and price-focused agents 
Figure 13 shows the mean average prices of the three different agents. The mean average price of the multi-attribute focused agent is 36.14 as compared with 22.2 (price-focused agent) and 52.61 (quality-focused agent). These numbers alone are not remarkable, but they become remarkable, if we also consider the average quality of the products in Figure 13. The price- and quality-focused DSS agent's average product quality is 6.4 in relation to 2.2 (price-focused agent) and 8.1 (quality-focused agent). The data shows that the intelligent multi-attribute focused agent buys products $31.3 \%$ cheaper but only with a $21.68 \%$ lower quality than the quality-focused agent. The price- and quality-focused agent buys products on an average of $62.81 \%$ more expensive but with a $191.14 \%$ better quality.

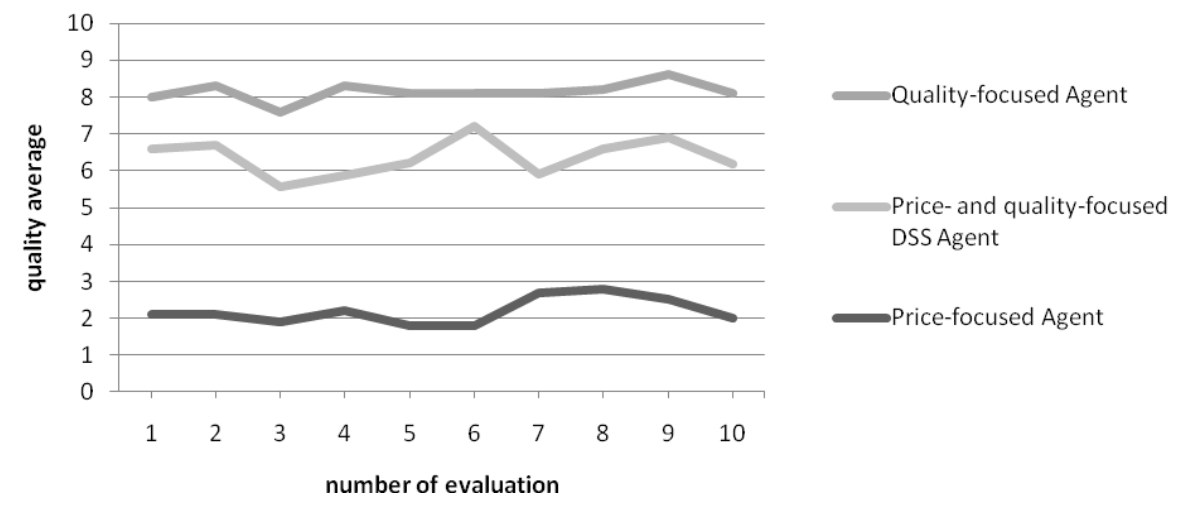

Fig. 13. Comparison between quality-, price-, and quality- and price-focused agents

The evaluations clearly demonstrated the advantage of an intelligent multi-attribute focused DSS agent in comparison to ordinary negotiation agents.

\section{Conclusion}

Most procurers want suppliers that can offer all of products and services defect-free, delivered just in time (or as close to this ideal as reasonably possible) and reliable. A supplier rating is needed to determine which suppliers are capable of coming satisfactorily close to this and thus to be retained as current suppliers. The procedure of a multifactor comparison, the combination of valuating the individual subjective criteria and the pricing and quality factors must often be done manually, which is error-prone and time intensive. Depending on the chosen level of complexity, usable tools range from spreadsheets to cost intensive and highly complex extensions of ERP-Systems, e.g. SAP. A solution supporting the user on the one hand in all automatable steps and on the other hand being flexible enough to implement the different methods of supplier rating is strongly needed, which at the same time should be achievable also for small and medium sized companies. Intelligent agent technology is recognized as a promising approach for enhancing a system's connectivity, extensibility, reusability and for dealing with its complexity, autonomy and heterogeneity. However, typically semantic gaps exist in the exploitation of current agent systems considering the fact that most times just syntactic matching is used for detecting equalities between exchanged data during the communication process. Ontologies can be used for representing concepts and relationships embedded in the negotiation environment so that they are semantically 
meaningful to a software agent, ensuring that agents refer to exactly the same object. In this chapter we combine multi-agent technology with ontology-driven solutions to build the Semantic-enabled Decision Support System, respectively SemDSS, which is able to support and partly automate procurement processes. We introduce semantic foundations to bridge relevant semantic gaps in order to radically improve the automated data processing in semantically heterogeneous environments. Our approach offers solutions for solving the interoperability problem in the procurement process.

Moreover, such a system increases the overall process efficiency and helps minimizing subjective judgment. In the last section, we presented the advantages when using SemDSS agents in automated negotiation. Using a history analysis and by slowly increasing its maximum offer after every lost auction, the DSS agent was able to win negotiations on an average lower price than the other negotiation agents. Besides, the SemDSS negotiation agents have shown benefits due to their ability to consider more than just one attribute of a negotiation.

Our future work will be concerned with the integration of the presented system in a real company framework as well as with the demonstration of the achieved results. Furthermore, we will focus on ontology merging and mapping, since it is complementary with our approach.

\section{References}

Aberdeen Group, The E-Procurement Benchmark report, August 2006.

Ai W.G., Sun J., and Li H., A distributed marketing decision support system based on multiintelligent-agent, Machine Learning and Cybernetics, Proceedings of 2004 International Conference, Aug. 2004, Vol 1, pp. 233-238.

Alvarado M., Sheremetov L., Bañares-Alcántara R., and Cantú-Ortiz F., Current challenges and trends in intelligent computing and knowledge management in industry, Knowl. Inf. Syst., vol. 12, 2007, S. 117-127.

Baclawski K., Kokar M., Kogut P., Hart L., Smith J., Holmes W., Letkowski J., Aronson M., (2001) Extending UML to Support Ontology Engineering for the Semantic Web, Proceedings of the 4th International Conference on UML, Springer, pp. 342-360

Beam C. and Segev A., Automated Negotiations: A Survey of the State of the Art, WIRTSCHAFTSINFORMATIK, vol. 39, 1997, pp. 263--268.

Bergamaschi S., Castano S., Vincini M., (1999) Semantic integration of semistruc-tured and structured data sources. SIGMOD Rec. 28, 1 (Mar. 1999), 54-59

Bianchini D., Antonellis V., and Melchiori M., Flexible Semantic-Based Service Matchmaking and Discovery, World Wide Web, vol. 11, 2008, S. 227-251.

Biffl, S., Sunindyo, W., and Moser, T., Semantic Integration of Heterogeneous Data Sources for Monitoring Frequent-Release Software Projects, in 4th International Conference on Complex, Intelligent and Software Intensive Systems (CISIS 2010), Krakow, Poland, 2010, pp. 360-367.

Bouzguenda L., Bouaziz R., and Andonoff E., Dynamic Plugging Of Business Processes In Cross-Organizational Workflow, International Journal of Computer Science and Applications, vol. 5, 2008, pp. 141-164.

Bui T. and Lee J., An agent-based framework for building decision support systems, Decis. Support Syst., vol. 25, 1999, S. 225-237.

Bundesverband für Materialwirtschaft, Einkauf und Logistik e. V., Marktstudie: Trends im Einkaufs-Outsourcing und eProcurement, 15.07.2003, Frankfurt 
Bruijn J., Kerrigan M., Zaremba M. and Fensel D.:Semantic Web Services, Staab S. and Studer R., (Eds), Handbook on Ontologies, Berlin, Heidelberg: Springer Berlin Heidelberg, 2009.

Calero C., Ruiz F., Piattin F., (2007) Ontologies for Software Engineering and Technology, Springer

Chandrasekaran B., Josephson J. R. and V. R Benjamins, (1999) What Are Ontologies, and Why Do We Need Them? IEEE Intelligent Systems. 1999

Cimiano P., Handschuh S., Staab S., (2004) Towards the Self-Annotating Web. In: Proceedings of the 13th International World Wide Web Conference, WWW 2004, New York, USA

David E., Azoulay-Schwartz R., and Kraus S., Bidding in sealed-bid and English multiattribute auctions, Decision Support Systems, vol. 42, Nov. 2006, pp. 527-556..

Davies J.. Semantic Web Technology, (2006) Trends and Research in Ontology-based Systems: Trends and Research. Wiley \& Sons

Eilbeck K., et al. (2005) The sequence ontology: a tool for the unification of genome annotations. Genome Biol. 6, R44

Fonseca F., Egenhofer M., Davis C., (2000) Ontology-Driven Information; The AAAI 2000 Workshop on Spatial and Temporal Granularity

Happel H.J., and Seedorf S., (2006) Applications of Ontologies in Software Engi-neering, Proceedings of the Workshop on Semantic Web Enabled Software Engineering (SWESE)

He, M., Jennings, N. R. and Leung, H. (2003) On agent-mediated electronic commerce. IEEE Trans on Knowledge and Data Engineering, 15 (4). pp. 985-1003.

Hepp M., De Leenheer P., De Moor A., Sure Y., (2007) Ontology Management. Semantic Web, Semantic Web Services, and Business Applications, Springer

Hikkanen A., Kalakota R., Saengcharoenrat P., Stallaert J., Whinston A.B., Distributed decision support systems for real time supply chain management using agent technologies, Readings in Electronic Commerce (1997) 275-291.

Horrocks, I. and Li L.: A Software Framework for Matchmaking Based on Semantic Web Technology. International Journal of Electronic Commerce. Vol. 8, Iss. 4, 39--60 (2004)

Houari N., and Far B.H., An intelligent project lifecycle data mart-based decision support system, Electrical and Computer Engineering, Canadian Conference, 2-5 May 2004, Vol 2, pp. 727-730.

Jain V, Wadhwa S, Deshmukh SG. 2009. Revisiting information systems to support a dynamic supply chain: issues and perspectives. Production Planning $\mathcal{E}$ Control: The Management of Operations 20(1):17-29.

Jennings N.R., An agent-based approach for building complex software systems, Commun. ACM, vol. 44, 2001, pp. 35-41.

Kersten G.E. and Lai H., Negotiation Support and E-negotiation Systems: An Overview, Group Decision and Negotiation, vol. 16, 2007, S. 553-586.

Koppensteiner G., Merdan M., Hegny I., and Weidenhausen G., A change-directionalgorithm for distributed multi-agent transport systems, Mechatronics and Automation, 2008. ICMA 2008. IEEE International Conference on, 2008, pp. 1030-1034.

Koppensteiner G., Merdan M., Lepuschitz W., Vittori L. and List E., Ontology-oriented Framework for Virtual Enterprises. International Conference on KnowledgeEngineering and Ontology-Management, October 6 - 8, Madeira, 2009, Portugal

Koppensteiner G., Merdan M., Lepuschitz W., Reinprecht C., Riemer R., Strobl S.: 2009a, A Decision Support Algorithm for Ontology-Based Negotiation Agents within Virtual Enterprises;: Future Information Technology and Management Engineering FITME 2009, Sanya, China; 
Kiryakov A., Popov B., Terziev I., Manov D., Ognyanoff D., (2004) Semantic annotation, indexing, and retrieval, Web Semantics: Science, Services and Agents on the World Wide WebVolume 2, Issue 1, December 2004, Pages 49-79

Kowalczyk, R., Ulieru, M., Unland, R.: Integrating Mobile and Intelligent Agents in Advanced E-commerce: A Survey. In: Agent Technologies, Infrastructures, Tools, and Applications for E-Services, Proceedings NODe'2002 Agent-Related Workshops, Erfurt, Germany. LNAI 2592, Springer-Verlag, pp.295-313, 2002.

Kuikka S., A batch process management framework: Domain-specific, design pattern and software component based approach, $\mathrm{PhD}$ thesis. Helsinki University of Technology, 1999. , 15

Lee C., Lau H., Ho G., and Ho W., Design and development of agent-based procurement system to enhance business intelligence, Expert Systems with Applications, vol. 36, Jan. 2009, S. 877-884.

Luke S. and Rager D., Ontology-Based Knowledge Discovery on the World-Wide Web, The 13th National Conference on Artificial Intelligence (AAAI96), 1996, S. 96--102.

Mäkelä, E.: Survey of semantic search research. In: Proc. of the Seminar on Knowledge Management on the Semantic Web (2005)

Merdan M., Zoitl A., Koppensteiner G., Demmelmayr F., Semantische Technologien - Stand der Technik, e\&i Elektrotechnik und Informationstechnik, IN PRESS

Merdan M., Knowledge-based Multi-Agent Architecture Applied in the Assembly Domain, PhD Thesis, Vienna University of Technology, 2009.,

Merdan M., Koppensteiner G., Hegny I., and Favre-Bulle B., Application of an ontology in a transport domain, IEEE International Conference on Industrial Technology, 2008. ICIT 2008, 2008, pp. 1-6., 20

Michelson M. and Knoblock C. A., (2007) An Automatic Approach to Semantic Annotation of Unstructured, Ungrammatical Sources: A First Look, In Proceedings of the 1st IJCAI Workshop on Analytics for Noisy Unstructured Text Data (AND-07), Hyderabad, India

Milanovic N. and Malek M., Current solutions for Web service composition, Internet Computing, IEEE, vol. 8, 2004, S. 51-59.

Miller G. A., (1995) WordNet: a lexical database for English, Communications of the ACM, v.38 n.11, p.39-41

Moser T., Semantic Integration of Engineering Environments Using an Engineering Knowledge Base, PhD Thesis, Vienna University of Technology, 2009.

Moser, T.; Biffl, S.; Sunindyo, W., and Winkler, D. Integrating Production Automation Expert Knowledge Across Engineering Stakeholder Domains Proc. 4th International Conference on Complex, Intelligent and Software Intensive Systems (CISIS 2010), IEEE Computer Society, 2010, 352-359.

Neubert R., Gorlitz O., Teich T., 2004.Automated negotiations of supply contracts for flexible production networks. International Journal of Production Economics 89(2), pp. 175-187.

Pastor Ó., España S. and González A., (2008) An Ontological-Based Ap-proach to Analyze Software Production Methods, Information Systems and e-Business Technologies, 2nd International United Information Systems Conference UNISCON 2008 Klagenfurt, Austria, April 22-25, Proceedings, Vol. 5, pp. 258-270

Preist C., Jennings N.R., Bartolini C., and Bartolini C., A Generic Software Framework for Automated Negotiation, IN: PROCEEDINGS OF SELMAS'2004, LNCS 3390, vol. 2004, 2002, pp. 213--235. 
Puigjaner L.,Guillen-Gosalbez G., Mele F.D.,2008. Multiagent framework for modeling and design of supply chain networks. In: Papageorgiou, Lazaros G., Georgiadis, MichaelC. (Eds.), Supply Chain Optimization, Part1. Wiley-VCH, pp. 45-83.

Rao J. and Su X., A Survey of Automated Web Service Composition Methods, In Proceedings of the First International Workshop on Semantic Web Services and Web Process Composition, SWSWPC 2004, 2004, S. 43--54.

Renna P. and Argoneto P., Production planning and automated negotiation for SMEs: An agent based e-procurement application, International Journal of Production Economics, vol. 127, Sep. 2010, S. 73-84.

Rinderle, S. and Benyoucef, M. (2005) Towards the Automation of E-Negotiation Processes Based on Web Services - A Modeling Approach. In: Proc. Int'l Conf. on Web Information Systems Engineering (WISE'05), New York.

Rouwenhorst B., Reuter B., Stockrahm V., van Houtum G.J., Mantel R.J., and Zijm W.H.M., Warehouse design and control: Framework and literature review, European Journal of Operational Research, vol. 122, May. 2000, pp. 515-533.,

Staab S., Maedche A., Handschuh S., (2001) An Annotation Framework for the Semantic Web, In: S. Ishizaki (ed.), Proc. of The First International Workshop on Multi-Media Annotation. January. 30 - 31, Tokyo, Japan

Sünder C., Zoitl A., and Dutzler C., Functional structure-based modelling of automation systems, in Int. J. Manufacturing Research, vol. 1, iss. 4, pp. 405-420, 2006. 16

Sycara K., Paolucci M., Ankolekar A., and Srinivasan N., Automated discovery, interaction and composition of Semantic Web services, Web Semantics: Science, Services and Agents on the World Wide Web, vol. 1, Dez. 2003, S. 27-46.

Tamma V., Phelps S., Dickinson I., and Wooldridge M., Ontologies for supporting negotiation in e-commerce, Engineering Applications of Artificial Intelligence, vol. 18, Mar. 2005, pp. 223-236.

Tamma V., Wooldridge M., Blacoe I., and Dickinson I., An Ontology Based Approach to Automated Negotiation, Agent-Mediated Electronic Commerce IV. Designing Mechanisms and Systems, 2002, pp. 317-334.

Turban, E., Aronson, J. E., and Liang, T. (2005) Decision Support Systems and Intelligent Systems (7th edition), Prentice Hall Publication, pp. 223 and pp. 109, 707.

Van den Berg J.P., A literature survey on planning and control of warehousing systems, IIE Transactions, vol. 31, 1999, pp. 751-762.,

Wang X.F., Chen Y.Q., and Liu Y.L., Web-oriented warfare command decision support system based on agent and data warehouse, Cyberworlds, 2005, International Conference, 23-25 Nov. 2005, pp. 6-11.

Yuan Y., Liang T.P., Zhang J.J., Using Agent Technology to Support Supply Chain Management: Potentials and Challenges,Working Paper, University Hamilton, 2002.

Zhang H. Application of Multi-agent Technology to Information Systems: An Agent-based Design Architecture for Decision Support Systems, Australasian Journal of Information Systems, Vol 15, No 2 (2009)

Zhang Q., and Yu J.H., Bidding Decision-Making Support System for Power Supplier Based on Multi-Agent System, Power System Technology, PowerCon 2006. International Conference, Oct. 2006, pp. 1-6.

Zhang Z.Q., and Xi B., Research on Framework of Decision Support System for Partner Choice and Measurement in Supply Chain, Machine Learning and Cybernetics, Proceedings of 2005 International Conference, 18-21 Aug. 2005, Vol 1, pp. 378-382. 


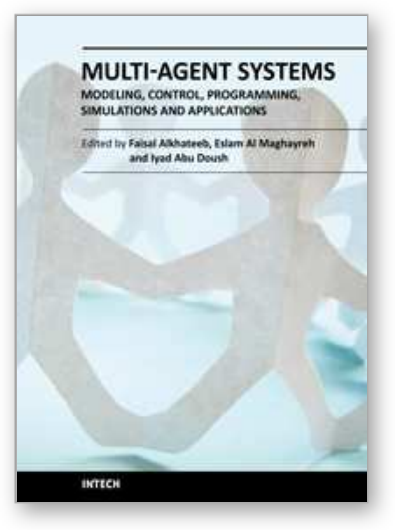

\author{
Multi-Agent Systems - Modeling, Control, Programming, \\ Simulations and Applications \\ Edited by Dr. Faisal Alkhateeb
}

ISBN 978-953-307-174-9

Hard cover, 522 pages

Publisher InTech

Published online 01, April, 2011

Published in print edition April, 2011

A multi-agent system (MAS) is a system composed of multiple interacting intelligent agents. Multi-agent systems can be used to solve problems which are difficult or impossible for an individual agent or monolithic system to solve. Agent systems are open and extensible systems that allow for the deployment of autonomous and proactive software components. Multi-agent systems have been brought up and used in several application domains.

\title{
How to reference
}

In order to correctly reference this scholarly work, feel free to copy and paste the following:

Koppensteiner Gottfried, Merdan Munir, Lepuschitz Wilfried, Moser Thomas and Reinprecht Constantin (2011). Multi Agent Systems Combined with Semantic Technologies for Automated Negotiation in Virtual Enterprises, Multi-Agent Systems - Modeling, Control, Programming, Simulations and Applications, Dr. Faisal Alkhateeb (Ed.), ISBN: 978-953-307-174-9, InTech, Available from: http://www.intechopen.com/books/multiagent-systems-modeling-control-programming-simulations-and-applications/multi-agent-systems-combinedwith-semantic-technologies-for-automated-negotiation-in-virtual-enterpr

\section{INTECH}

open science | open minds

\section{InTech Europe}

University Campus STeP Ri

Slavka Krautzeka 83/A

51000 Rijeka, Croatia

Phone: +385 (51) 770447

Fax: +385 (51) 686166

www.intechopen.com

\section{InTech China}

Unit 405, Office Block, Hotel Equatorial Shanghai

No.65, Yan An Road (West), Shanghai, 200040, China

中国上海市延安西路65号上海国际贵都大饭店办公楼 405 单元

Phone: +86-21-62489820

Fax: $+86-21-62489821$ 
(C) 2011 The Author(s). Licensee IntechOpen. This chapter is distributed under the terms of the Creative Commons Attribution-NonCommercialShareAlike-3.0 License, which permits use, distribution and reproduction for non-commercial purposes, provided the original is properly cited and derivative works building on this content are distributed under the same license. 